\title{
Modeling a nonperturbative spinor vacuum interacting with a strong gravitational wave
}

\author{
Vladimir Dzhunushaliev ${ }^{1,2, a} \mathbb{D}$, Vladimir Folomeev ${ }^{3, b}$ \\ ${ }^{1}$ Department of Theoretical and Nuclear Physics, Al-Farabi Kazakh National University, Almaty 050040, Kazakhstan \\ 2 Institute of Experimental and Theoretical Physics, Al-Farabi Kazakh National University, Almaty 050040, Kazakhstan \\ ${ }^{3}$ Institute of Physicotechnical Problems and Material Science of the NAS of the Kyrgyz Republic, 265 a, Chui Street, Bishkek 720071, \\ Kyrgyz Republic
}

Received: 27 March 2015 / Accepted: 17 June 2015 / Published online: 4 July 2015

(C) The Author(s) 2015. This article is published with open access at Springerlink.com

\begin{abstract}
We consider the propagation of strong gravitational waves interacting with a nonperturbative vacuum of spinor fields. To described the latter, we suggest an approximate model. The corresponding Einstein equation has the form of the Schrödinger equation. Its gravitational-wave solution is analogous to the solution of the Schrödinger equation for an electron moving in a periodic potential. The general solution for the periodic gravitational waves is found. The analog of the Kronig-Penney model for gravitational waves is considered. It is shown that the suggested gravitational-wave model permits the existence of weak electric charge and current densities concomitant with the gravitational wave. Based on this observation, a possible experimental verification of the model is suggested.
\end{abstract}

\section{Introduction}

An experimental search for gravitational waves (GW) is one of the most intriguing problems in modern physics. The discovery of GWs will give us confidence that we are moving in the right direction in understanding classical gravity. If we cannot detect GWs, then either our apparatus is not sensitive enough or something is not taken into account when considering the propagation of GWs.

One of the effects accompanying the propagation of GWs could be its interaction with a nonperturbative spinor vacuum. The reason for such an interaction to occur is that the energy-momentum tensor of a spinor field contains the spin connection, which in turn contains first derivatives of tetrad components with respect to the coordinates. As a result, the Einstein equations give the wave equation for a $\mathrm{GW}$ which

\footnotetext{
a e-mail: v.dzhunushaliev@ gmail.com

b e-mail: vfolomeev@mail.ru
}

contains second derivatives of the tetrad components on the left-hand side and their first derivatives on the right-hand side.

In Ref. [1] we have considered the propagation of a weak $\mathrm{GW}$ interacting with the nonperturbative spinor vacuum. Here we extend those results to the case of a strong GW. In doing so, as in Ref. [1], to model the nonperturbative vacuum of a spinor field, we will use a phenomenological approach. Within the framework of this approach, we make some physically reasonable assumptions as regards expectation values of the spinor field and its dispersion. This will permit us to reduce the infinite system of differential equations for all Green functions of the nonperturbative quantum spinor field to the finite set of equations (for more details, see Refs. [1,2]).

Following this approach, here we will discuss the solution of the Einstein equations for a strong GW propagating on the background of the nonperturbative vacuum of spinor fields, which is a generalization of the weak, plane gravitational wave of Ref. [1].

\section{Strong GW in a nonperturbative spinor vacuum}

According to the textbook [3], the metric for a strong GW propagating in one direction is sought in the form

$\mathrm{d} s^{2}=2 \mathrm{~d} \xi \mathrm{d} \eta+g_{a b}(\eta) \mathrm{d} x^{a} \mathrm{~d} x^{b}$,

where $x^{0}=\xi, x^{1}=\eta$ are lightlike coordinates, and the indices $a, b$ run over the values 2,3 . It is convenient to introduce the new variables

$g_{a b}(\eta)=-\chi^{2}(\eta) \gamma_{a b}(\eta)$,
$\operatorname{det}\left(\gamma_{a b}\right)=1$. 
The only nonvanishing component of the Einstein tensor $G_{\bar{\mu}} \nu$ is $G_{\overline{1} \eta}(\bar{\mu}$ is the tetrad index and $v$ is the spacetime index), so that one can obtain the following Einstein equation for the strong GW propagating in a flat vacuum spacetime:

$\ddot{\chi}+\frac{1}{8}\left(\dot{\gamma}_{a c} \gamma^{b c} \dot{\gamma}_{b d} \gamma^{a b}\right) \chi=0$.

Here the dot denotes differentiation with respect to $\eta ; \gamma^{a b}$ is the two-dimensional tensor reciprocal to $\gamma_{a b}$. The function $\chi(\eta)$ is an unknown function, and $\gamma_{a b}$ are arbitrary functions, obeying the constraint (3).

As pointed out in Ref. [3], the presence of the term $\dot{\gamma}_{a c} \gamma^{b c} \dot{\gamma}_{b d} \gamma^{a b}$ has the result that after a finite time interval $\chi$ becomes zero.

This in turn leads to vanishing of the metric determinant $g$, i.e., a singularity in the metric. But this singularity is not physically significant since it is related only to the unsatisfactory nature of the reference frame, "spoiled" by the passing gravitational wave, and can be eliminated by a suitable coordinate transformation.

Our goal here is to consider the propagation of GWs in a nonperturbative spinor vacuum. We expect that such a physical system has to be considered in nonperturbative language when both a metric and a spinor field are regarded as quantum quantities and are quantized in a nonperturbative manner. Then, to describe the interaction between a quantum metric and a quantum spinor field, we have to write down the Einstein-Dirac operator equations.

Our nonperturbative approach for quantizing nonlinear fields is described in Ref. [1]. Within this method, we have, strictly speaking, to solve the following operator equations:

$\hat{G}_{\bar{a} \mu}=\varkappa \hat{T}_{\bar{a}}$,

$\hat{\gamma}^{\mu} \nabla_{\mu} \hat{\psi}-m \hat{\psi}=0$,

where $\hat{G}_{\mu \nu}$ is the operator of the Einstein tensor; $\hat{T}_{\mu \nu}$ is the operator of the energy-momentum tensor; $\hat{\psi}$ is the operator of the spinor field; $\bar{a}$ is the tetrad index; $\mu$ is the coordinate index; $\nabla_{\mu}$ is the covariant derivative for the spinor with the appropriate spin connection; $\varkappa=8 \pi G, G$ is the gravitational constant. Hereafter we use units where $c=\hbar=1$.

As mentioned in Ref. [1], the solution for such a set of operator equations can be found by writing down and subsequent solving an infinite system of equations for all Green functions for quantum fields.

In practice, such a procedure can be carried out only approximately. This means that we have to use some physical arguments to cut off the above-mentioned infinite set of equations, and then solve the resulting finite set of equations.

Our approach is based on the following assumptions:

(i) The metric remains always classical.

(ii) The spinor field is quantum. (iii) The spinor field is decomposed as a product of $q$ - and $c$-numbers.

(iv) In order to calculate the energy-momentum tensor of the spinor field, we assume some ansätze for a 2-point Green function of $\hat{\psi}$.

In such an approximation the Einstein-Dirac operator equations (5) and (6) can be written in the following manner [1]:

$$
\begin{gathered}
G_{\bar{a} \mu}=\varkappa\left\langle Q\left|\hat{T}_{\bar{a} \mu}\right| Q\right\rangle, \\
\left\langle Q\left|\nabla_{\mu} \hat{T}_{\bar{a}}{ }^{\mu}\right| Q\right\rangle=0,
\end{gathered}
$$

where $\langle Q|\cdots| Q\rangle$ is the quantum averaging with respect to the quantum state $|Q\rangle$.

Also, to check the consistency of the ansätze for a 2-point Green function, instead of solving the Dirac equation (6), we will use the Bianchi identities (8), as we did in Ref. [1].

\section{Approximate model of the nonperturbative spinor vacuum}

In order to write down the energy-momentum tensor for a vacuum of a spinor field interacting with a $\mathrm{GW}$, we must have a model of a nonperturbative vacuum of the spinor field. Let us emphasize once more that we cannot use a perturbative model of a spinor field since in the presence of a gravitational field the set of Eqs. (5) and (6) is a strongly nonlinear system.

Taking this into account and following Ref. [1], our strategy in the formulation of the model of the nonperturbative spinor vacuum is as follows: (i) we write some classical ansätze for a spinor; (ii) we derive the corresponding energymomentum tensor; and (iii) we then write hats over the corresponding spinor components.

As the first step, we take the ansätze for the classical spinor in the form

$\psi=e^{i \omega \eta}\left(\begin{array}{l}A \\ B \\ V \\ Q\end{array}\right)$.

For the tetrad (13) below, there is only one nonvanishing component $G_{\overline{1} \eta}$. Consequently, we have to choose the components of the spinor $A, B, V, Q$ so that we obtain the corresponding component $T_{\overline{1} \eta}$. This happens if $B=A$ and $Q=V$. In this case we have

$T_{\overline{1} \eta}=-2 V V^{*}\left(\beta^{\prime} \cosh \beta+\alpha^{\prime} \sinh \beta+4 \omega\right)$,

where the prime denotes $d / d \eta$. 
With the ansätze (9) and the energy-momentum tensor (10) in hand, we suggest the following approximate model of the nonperturbative vacuum of the spinor field.

- The nonperturbative vacuum is described by the following operator of the spinor field:

$$
\hat{\psi}=e^{i \omega \eta}\left(\begin{array}{c}
\hat{A} \\
\hat{A} \\
\hat{V} \\
\hat{V}
\end{array}\right)
$$

The constant operators $\hat{A}, \hat{V}$ appearing here are independent of $\eta$.

- The corresponding energy-momentum tensor of the spinor field is

$$
\left\langle Q\left|\hat{T}_{\overline{1 \eta}}\right| Q\right\rangle=-2\left\langle\hat{V} \hat{V}^{\dagger}\right\rangle\left(\beta^{\prime} \cosh \beta+\alpha^{\prime} \sinh \beta+4 \omega\right) .
$$

- To check this model, we calculate the divergence of the energy-momentum tensor and show that it vanishes.

\section{Einstein equations for a strong GW interacting with the nonperturbative spinor vacuum}

For the metric (1), we seek a solution of the Einstein equations for a strong GW propagating through the nonperturbative spinor vacuum. The tetrad for this metric is

$e_{\mu}^{\bar{a}}=\left(\begin{array}{cccc}0 & 1 & 0 & 0 \\ 1 & 0 & 0 & 0 \\ 0 & 0 & \chi(\eta) e^{\alpha(\eta) / 2} & 0 \\ 0 & 0 & \chi(\eta) e^{\alpha(\eta) / 2} \sinh \beta(\eta) & \chi(\eta) e^{-\alpha(\eta) / 2}\end{array}\right)$

with the corresponding two-dimensional metric

$\gamma_{a b}=\left(\begin{array}{cc}e^{\alpha} \cosh ^{2} \beta & \sinh \beta \\ \sinh \beta & e^{-\alpha}\end{array}\right)$.

Here $\alpha(\eta)$ and $\beta(\eta)$ are arbitrary functions [3].

The substitution of the tetrad (13) and the energymomentum tensor (12) into the Einstein equations (7) yields the equation

$$
\begin{gathered}
-\chi^{\prime \prime}+\left[\beta^{\prime} \cosh \beta+\alpha^{\prime} \sinh \beta-\frac{1}{4}\left(\alpha^{\prime 2}+{\beta^{\prime}}^{2}\right) \cosh ^{2} \beta\right. \\
\left.-\frac{1}{4} \alpha^{\prime} \beta^{\prime} \sinh 2 \beta\right] \chi=-4 \tilde{\omega} \chi
\end{gathered}
$$

where the prime denotes differentiation with respect to the dimensionless $\tilde{\eta}=\varkappa\left\langle\hat{V} \hat{V}^{\dagger}\right\rangle \eta$, and the dimensionless $\tilde{\omega}=\omega /\left(\varkappa\left\langle\hat{V} \hat{V}^{\dagger}\right\rangle\right)$. (For convenience, we omit the tilde from $\tilde{\eta}$ and $\tilde{\omega}$ hereafter.) The terms $\beta^{\prime} \cosh \beta, \alpha^{\prime} \sinh \beta$ on the left-hand side and $4 \omega$ on the right-hand side of Eq. (15) are the imprints of the nonperturbative spinor vacuum.

One sees immediately that Eq. (15) is a Schrödinger-like equation with the effective potential

$$
\begin{aligned}
V_{\mathrm{eff}}= & \beta^{\prime} \cosh \beta+\alpha^{\prime} \sinh \beta-\frac{1}{4}\left(\alpha^{\prime 2}+{\beta^{\prime}}^{2}\right) \cosh ^{2} \beta \\
& -\frac{1}{4} \alpha^{\prime} \beta^{\prime} \sinh 2 \beta
\end{aligned}
$$

In what follows we will seek periodic solutions to Eq. (15). It is clear that for periodic functions $\alpha, \beta$ we obtain an equation similar to that describing the movement of a single electron in a crystal. Such an equation has been well studied in the literature (see, for example, the textbook [4]), and we can apply all mathematical methods used in solving the Schrödinger equation to our case of a strong GW.

\section{General solution}

In order to solve Eq. (15) for a periodic potential, in this section we apply the methods developed in solid state theory. The only difference is that the function $\chi(\eta)$ is a real function, unlike the usual quantum mechanics where a wave function is complex.

First of all, let us rewrite Eq. (15) in the form

$-\chi^{\prime \prime}(\eta)+V_{\mathrm{eff}}(\eta) \chi(\eta)=-4 \omega \chi(\eta)$

In order to find a solution of the type of $\mathrm{GW}$, we have to investigate the case of a periodic potential

$V_{\text {eff }}\left(\eta+\eta_{0}\right)=V_{\text {eff }}(\eta)$

where $\eta_{0}$ is the period of the effective potential.

We seek a solution to Eq. (17) in the form

$\chi(\eta)=\chi_{0}+\sum_{k=1}^{\infty}\left[a_{k} \cos \left(\frac{2 \pi k}{\eta_{0}} \eta\right)+b_{k} \sin \left(\frac{2 \pi k}{\eta_{0}} \eta\right)\right]$.

In order to obtain a set of equations for the coefficients $\chi_{0}, a_{k}, b_{k}$, we act as follows: (a) integrate Eq. (17); (b) multiply (17) by $\frac{2}{\eta_{0}} \cos \left(\frac{2 \pi n}{\eta_{0}} \eta\right)$ and integrate; and (c) multiply (17) by $\frac{2}{\eta_{0}} \sin \left(\frac{2 \pi n}{\eta_{0}} \eta\right)$ and integrate. This yields the following system of equations: 


$$
\begin{aligned}
& V_{0}+\frac{1}{2} \sum_{k=1}^{\infty}\left[a_{k} \tilde{V}_{k}+b_{k} \bar{V}_{k}\right]=-4 \omega \chi_{0} \\
& \left(\frac{2 \pi n}{\eta_{0}}\right)^{2} a_{n}+\chi_{0} \tilde{V}_{n}+\sum_{k=1}^{\infty}\left[a_{k} \tilde{V}_{n k}+b_{k} \bar{V}_{n k}\right]=-4 \omega a_{n}, \\
& \left(\frac{2 \pi n}{\eta_{0}}\right)^{2} b_{n}+\chi_{0} \bar{V}_{n}+\sum_{k=1}^{\infty}\left[a_{k} \bar{V}_{k n}+b_{k} \tilde{\tilde{V}}_{n k}\right]=-4 \omega b_{n},
\end{aligned}
$$

where $n=1,2, \ldots, \infty$ and

$$
\begin{aligned}
& V_{0}=\frac{\chi_{0}}{\eta_{0}} \int_{-\eta_{0} / 2}^{+\eta_{0} / 2} V_{\mathrm{eff}} \mathrm{d} \eta ; \\
& \tilde{V}_{k}=\frac{2}{\eta_{0}} \int_{-\eta_{0} / 2}^{+\eta_{0} / 2} V_{\mathrm{eff}} \cos \left(\frac{2 \pi k}{\eta_{0}} \eta\right) \mathrm{d} \eta, k=1,2, \ldots, \infty ; \\
& \bar{V}_{k}=\frac{2}{\eta_{0}} \int_{-\eta_{0} / 2}^{+\eta_{0} / 2} V_{\mathrm{eff}} \sin \left(\frac{2 \pi k}{\eta_{0}} \eta\right) \mathrm{d} \eta, k=1,2, \ldots, \infty ; \\
& \tilde{V}_{n k}=\frac{2}{\eta_{0}} \int_{-\eta_{0} / 2}^{+\eta_{0} / 2} \cos \left(\frac{2 \pi n}{\eta_{0}} \eta\right) V_{\mathrm{eff}} \cos \left(\frac{2 \pi k}{\eta_{0}} \eta\right) \\
& \bar{V}_{n k}=\frac{2}{\eta_{0}} \int_{-\eta_{0} / 2}^{+\eta_{0} / 2} \cos \left(\frac{2 \pi n}{\eta_{0}} \eta\right) V_{\mathrm{eff}} \sin \left(\frac{2 \pi k}{\eta_{0}} \eta\right) \\
& \mathrm{d} \eta, \quad, k=1,2, \ldots, \infty ; \\
& \tilde{\tilde{V}}_{n k}=\frac{2}{\eta_{0}} \int_{-\eta_{0} / 2}^{+\eta_{0} / 2} \sin \left(\frac{2 \pi n}{\eta_{0}} \eta\right) \\
& V_{\mathrm{eff}} \sin \left(\frac{2 \pi k}{\eta_{0}} \eta\right) \mathrm{d} \eta, \quad n, k=1,2, \ldots, \infty .
\end{aligned}
$$

In principle, by using the set of Eqs. (20)-(22), one can find a GW solution for any periodic metric functions $\alpha(\eta)$ and $\beta(\eta)$. Remarkably, in doing so, one can employ all welldeveloped methods of solid state theory (see, for example, the textbook [4]).

\section{The Kronig-Penney model for gravitational waves}

In the previous section we have used the well-known methods of solid state theory to obtain the general solution for the GW. To discuss some particular features of the GW, here we employ an approximate approach of solid state theory (the Kronig-Penney model) for the description of the GW.
Let us consider the simplest case, $\beta=0$, for which Eq. (17) yields

$-\chi^{\prime \prime}-\frac{1}{4} \alpha^{\prime 2} \chi=-4 \omega \chi$.

For such a case we have the following two-dimensional metric (14):

$\gamma_{a b}=\left(\begin{array}{cc}e^{\alpha} & 0 \\ 0 & e^{-\alpha}\end{array}\right)$

In order to apply the Kronig-Penney approximation, we will use the metric function $\alpha(\eta)$ in the range $-a<\eta<b$ :

$\alpha(\eta)= \begin{cases}C_{0} & \text { if }-a<\eta<0, \\ V_{0} \eta+C_{1} & \text { if } 0<\eta<\frac{b}{2}, \\ -V_{0} \eta+C_{2} & \text { if } \frac{b}{2}<\eta<b,\end{cases}$

where $C_{0,1,2}$ and $V_{0}$ are constants; $a+b=\eta_{0}$, and

$\alpha\left(\eta+n \eta_{0}\right)=\alpha(\eta), \quad n= \pm 1, \pm 2, \ldots$

The corresponding effective potential $V_{\text {eff }}=-\alpha^{\prime 2} / 4$ is

$V_{\text {eff }}= \begin{cases}0 & \text { if }-a<\eta<0, \\ -\frac{V_{0}^{2}}{4} & \text { if } \quad 0<\eta<b\end{cases}$

with the periodicity

$V_{\text {eff }}\left(\eta+n \eta_{0}\right)=V_{\text {eff }}(\eta), \quad n= \pm 1, \pm 2, \ldots$

The profiles of $\alpha$ and $V_{\text {eff }}$ are shown in Fig. 1.

To solve Eq. (29) with the effective potential (33), we will employ the Kronig-Penney method exactly in the same way as in solid state theory but remembering that we have to seek a real solution instead of a complex one, as is done for the Schrödinger equation.

For the periodic potential (33), Eq. (29) takes the form

$$
\begin{cases}\chi^{\prime \prime}=K^{2} \chi & \text { if }-a<\eta<0 \\ \chi^{\prime \prime}=-Q^{2} \chi & \text { if } \quad 0<\eta<b\end{cases}
$$

where $K^{2}=4 \omega, Q^{2}=V_{0}^{2} / 4-K^{2}$.

For the fundamental region $-a \leq \eta \leq b$, we seek a solution in the following form:

$\chi(\eta)= \begin{cases}f e^{K \eta}+g e^{-K \eta} & \text { if }-a<\eta<0, \\ c \sin Q \eta+d \cos Q \eta & \text { if } 0<\eta<b .\end{cases}$ 


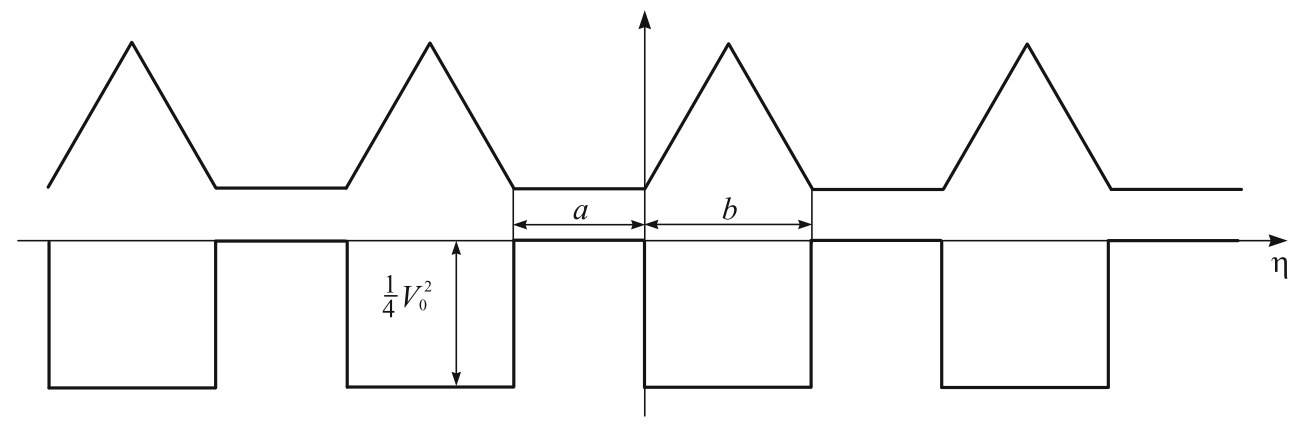

Fig. 1 The profiles of $\alpha(\eta)$ (the top graph) and $V_{\text {eff }}=-\frac{\alpha^{\prime 2}(\eta)}{4}$ (the bottom graph)

Using these, we first constrain continuity inside the fundamental domain (i.e., for $\eta=0$ ):

$\left\{\begin{array}{l}f+g=d, \\ K(f-g)=Q c .\end{array}\right.$

For the region $b \leq \eta \leq a+b$, we seek a solution in the form

$\chi(\eta)=f e^{K[\eta-(a+b)]}+g e^{-K[\eta-(a+b)]}$.

We then constrain continuity outside the fundamental domain (i.e., for $\eta=b$ ):

$\left\{\begin{array}{l}c \sin Q b+d \cos Q b=f e^{-K a}+g e^{K a}, \\ c Q \cos Q b-d Q \sin Q b=f K e^{-K a}-g K e^{K a} .\end{array}\right.$

A necessary condition for the set of Eqs. (37) and (39) to have a nontrivial solution is that the determinant of the corresponding matrix is zero:

$\left|\begin{array}{cccc}0 & 1 & -1 & -1 \\ Q & 0 & -K & K \\ \sin Q b & \cos Q b & -e^{-K a} & -e^{K a} \\ Q \cos Q b & -Q \sin Q b & -K e^{-K a} & K e^{K a}\end{array}\right|=0$.

The resulting constraint equation for the parameters $K, Q, a$, and $b$ is

$\frac{K^{2}-Q^{2}}{2 K Q} \sinh a K \sin b Q+\cosh a K \cos b Q=1$.

Introducing the dimensionless parameters

$x=\frac{4 \omega}{V_{0}^{2} / 4}, \quad \tilde{a}=\frac{a V_{0}}{2}, \quad \tilde{b}=\frac{b V_{0}}{2}$,

we obtain from (41)

$$
\begin{aligned}
& \frac{2 x-1}{2 \sqrt{x-x^{2}}} \sinh (\tilde{a} \sqrt{x}) \sin (\tilde{b} \sqrt{1-x}) \\
& \quad+\cosh (\tilde{a} \sqrt{x}) \cos (\tilde{b} \sqrt{1-x})=1 .
\end{aligned}
$$

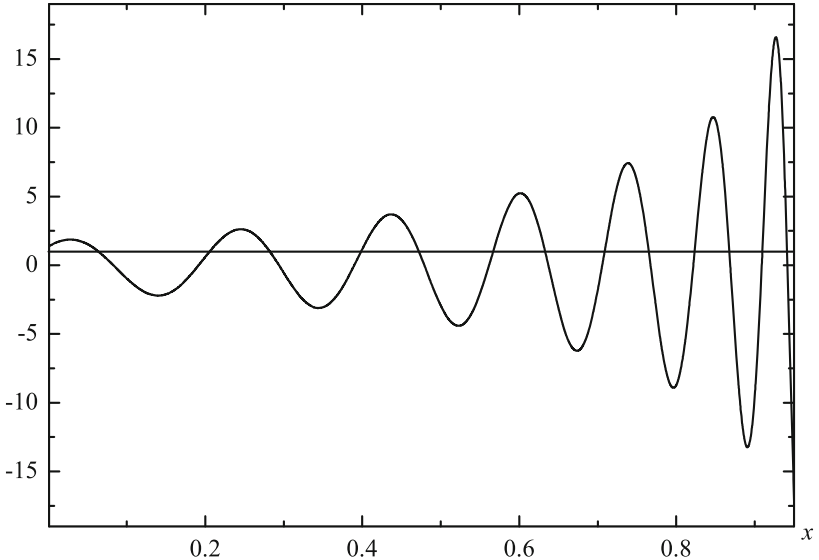

Fig. 2 The profile of the left-hand side of the constraint equation (43) for $\tilde{a}=3$ and $\tilde{b}=50$. The straight line corresponds to 1

Here $0<x \leq 1$, i.e., $0<\omega \leq V_{0}^{2} / 16$. The latter expression is obtained from the condition $Q^{2} \geq 0$, which has been assumed to be satisfied in the derivation of the above results. The case of $Q^{2}<0$ is discussed below.

The typical profile of the left-hand side (lhs) of the constraint equation (43) is shown in Fig. 2. One can see that there is a finite number $n$ of possible values of $\omega_{n}$, which depends on the values of the parameters $\tilde{a}$ and $\tilde{b}$. The numerical values of $\omega_{n}$ are determined by the points of intersection of the lines in Fig. 2. Note that in the limit $x \rightarrow 0$ the lhs $=-(\tilde{a} / 2) \sin \tilde{b}+\cos \tilde{b}$, and when $x=1$, the lhs $=(\tilde{b} / 2) \sinh \tilde{a}+\cosh \tilde{a}$, i.e., the lhs remains always finite.

The case of $Q^{2}<0$ can be obtained from (41) by changing $Q$ to $i Q$ :

$\frac{K^{2}+Q^{2}}{2 K Q} \sinh a K \sinh b Q+\cosh a K \cosh b Q=1$.

The corresponding dimensionless equation will then be

$$
\begin{aligned}
& \frac{2 x-1}{2 \sqrt{x^{2}-x}} \sinh (\tilde{a} \sqrt{x}) \sinh (\tilde{b} \sqrt{x-1}) \\
& +\cosh (\tilde{a} \sqrt{x}) \cosh (\tilde{b} \sqrt{x-1})=1 .
\end{aligned}
$$


Here $x>1$, i.e., $\omega>V_{0}^{2} / 16$. One can easily see that the lhs of this expression is always greater than 1, i.e., Eq. (45) does not have a solution. Physically, this means that there are no GWs with $\omega>V_{0}^{2} / 16$.

\section{Experimental verification}

Let us discuss now possible experimental consequences coming from a consideration of the propagation of a strong $\mathrm{GW}$ through the nonperturbative spinor vacuum.

Direct calculations show that for such a case the following electric current exists:

$j^{\mu}=\bar{\psi} \gamma^{\mu} \psi=\left(4\left\langle Q\left|\hat{V} \hat{V}^{\dagger}\right| Q\right\rangle, 0,0,0\right)$,

where $\mu=\xi, \eta, y, z$.

Making the transformation to Cartesian coordinates $t, x$ as follows:

$\xi=\frac{t-x}{\sqrt{2}}, \quad \eta=\frac{t+x}{\sqrt{2}}$

and similarly

$j^{\xi}=\frac{j^{t}-j^{x}}{\sqrt{2}}, \quad j^{\eta}=\frac{j^{t}+j^{x}}{\sqrt{2}}$,

we have

$j^{t}=-j^{x}=2 \sqrt{2}\left\langle Q\left|\hat{V} \hat{V}^{\dagger}\right| Q\right\rangle$.

This means that we have the electric charge and current densities concomitant with the GW. The electric current is directed along the direction of propagation of the GW. This observation allows us to suggest the following experimental verification of the $\mathrm{GW}$ and nonperturbative spinor vacuum models considered here: It is possible to try to measure the weak electric charge and current densities together with the standard measurements of GWs (LIGO, LISA, and so on). On the other hand, instead of measurements of the electric current, one can measure the corresponding magnetic field.

\section{The correctness of the ansätze for the spinor field}

The correctness of Eq. (8) is verified directly. To do this, one can calculate the expression $\nabla_{\mu} \hat{T}_{\bar{a}}{ }^{\mu}$ as the classical one and then substitute in it the 2-point Green function $\left\langle\hat{\psi}^{*} \hat{\psi}\right\rangle$. Then, taking into account that we have only one nonvanishing component of the energy-momentum tenor $T_{\overline{1} \eta}$ [see Eq. (10)], the Bianchi identities (8) are trivially satisfied.

\section{Conclusion}

To summarize our results:

- We have suggested an approximate model of the nonperturbative spinor vacuum.

- The propagation of strong gravitational waves interacting with such a vacuum has been investigated.

- It was shown that the Einstein equation reduces to a Schrödinger-like equation with a periodic potential.

- We have obtained the solution for the special case of the metric function corresponding to a $\mathrm{GW}$ with the $g_{y y, z z}$ components only.

- A possible experimental verification of the nonperturbative spinor vacuum model interacting with a strong gravitational wave has been suggested.

For better qualitative understanding of some features of the resulting gravitational waves, we have considered the simplest case where the periodic potential is modeled by the Kronig-Penney potential of solid state theory. As a result, it was shown that the GW parameters (the wavelength $\eta_{0}$ and the amplitude $V_{0}$ ) can be arbitrary but the parameter $\omega_{n}$, affecting the form of the plane wave in the spinor vacuum, is quantized.

The correctness of the approximate model of the nonperturbative spinor vacuum was verified by calculating the divergence of the right-hand side of the Einstein equations. One might expect that for an improved model of the nonperturbative vacuum it will be necessary to use the nonperturbatively quantized Dirac equation where the term "spin connection $\times$ spinor field" (i.e., $\left.\left\langle Q\left|\omega_{a b \mu} \psi\right| Q\right\rangle\right)$ need to be taken into account. The latter means that we have to take into account the quantum correlation between a metric and a spinor field.

Lastly, it may be noted that the formal analogy of the GW equation here obtained with the Schrödinger equation allows the possibility of using the well-developed methods from other fields of physics to solve such an equation. In particular, for the periodic metric functions discussed here, Eq. (17) is identical in form to the equation of motion of an electron in a crystal. Aside from the formal aspect, such an analogy would lead us to use physical intuition to obtain further results in this area, as is done, for example, in analogue gravity $[5,6]$.

Acknowledgments We acknowledge support from a grant No. 0263/PCF-14 in fundamental research in natural sciences by the Ministry of Education and Science of Kazakhstan.

Open Access This article is distributed under the terms of the Creative Commons Attribution 4.0 International License (http://creativecomm ons.org/licenses/by/4.0/), which permits unrestricted use, distribution, and reproduction in any medium, provided you give appropriate credit to the original author(s) and the source, provide a link to the Creative 
Commons license, and indicate if changes were made. Funded by SCOAP ${ }^{3}$.

\section{References}

1. V. Dzhunushaliev, V. Folomeev, Eur. Phys. J. C 74(9), 3057 (2014). arXiv:1405.6121 [gr-qc]
2. V. Dzhunushaliev, V. Folomeev, B. Kleihaus, J. Kunz, Eur. Phys. J. C 74, 2743 (2014). arXiv:1312.0225 [gr-qc]

3. L. Landau, E. Lifshitz, The Classical Theory of Fields (Pergamon, Oxford, 1987)

4. W.A. Harrison, Solid State Theory (Dover Publications, New York, 2011)

5. C. Barcelo, S. Liberati, M. Visser, Living Rev. Rel. 8, 12 (2005) arXiv:gr-qc/0505065

6. C. Barcelo, S. Barcelo, M. Visser, Living Rev. Rel. 14(3), 3 (2011) 